\title{
Flood routing modelling with Artificial Neural Networks
}

\author{
R. Peters, G. Schmitz, and J. Cullmann \\ Institute of Hydrology and Meteorology, University of Technology, Dresden, Germany \\ Received: 23 January 2006 - Revised: 22 May 2006 - Accepted: 3 July 2006 - Published: 26 September 2006
}

\begin{abstract}
For the modelling of the flood routing in the lower reaches of the Freiberger Mulde river and its tributaries the one-dimensional hydrodynamic modelling system HECRAS has been applied. Furthermore, this model was used to generate a database to train multilayer feedforward networks.

To guarantee numerical stability for the hydrodynamic modelling of some $60 \mathrm{~km}$ of streamcourse an adequate resolution in space requires very small calculation time steps, which are some two orders of magnitude smaller than the input data resolution. This leads to quite high computation requirements seriously restricting the application - especially when dealing with real time operations such as online flood forecasting.

In order to solve this problem we tested the application of Artificial Neural Networks (ANN). First studies show the ability of adequately trained multilayer feedforward networks (MLFN) to reproduce the model performance.
\end{abstract}

\section{Introduction}

Recent extreme flood events in central Europe, e.g. the flood of August 2002, which affected - amongst others - the catchment of the Freiberger Mulde river, led to an increased demand for fast and robust prediction tools.

The proper modelling of flood wave propagation faces challenges like backwater effects at river junctions and wide floodplains. To take this into account sophisticated hydrodynamic modelling is necessary. We applied the HEC-River Analysis System (HEC-RAS), which is a one-dimensional hydrodynamic model based on a numerical solution of the St-Venant-equations. This allows - and requires - the use of detailed topographical information representing the hydraulic properties of the river reaches. The river bed and the floodplains are described by geometrical data and roughness parameters of representative cross sections. Obviously, the accuracy of the model is related to the distance between the cross sections.

To avoid numerical instabilities a high resolution in space requires a small computation interval. This relationship is described by the Courant criterion. In this study a mean cross section distance of some $150 \mathrm{~m}$ corresponded to time steps of about 15 seconds. This leads to relatively high computational efforts. That is the reason why in case of real time operations the application of such a highly sophisticated model is not very functional. For this purpose fully robust and fast simulation tools like ANN is needed.

Previous studies concerning the application of ANN for the simulation of routing processes only used observed data. Shrestha et al. (2005) trained MLFN for the purpose of flood flow simulation at the Neckar river using a hydrodynamic numerical model, but only to provide data for unobserved locations for historical flood events. The main problem remains the lack of extrapolation capability. Or, as stated by Minns and Hall (1996): "ANN are a prisoner of their training data". We used hydrodynamic modelling to generate a database for the training of the MLFN covering the whole range of possible flood events. This guaranties the capability of the trained MLFN to predict extreme events beyond recorded floods.

The methodology was successfully tested at the lower river reaches of the Freiberger Mulde catchment (Fig. 1).

\section{Methodology}

A general description of the methodology in the form of a flow chart is showed in Fig. 2. In the following the particular steps will be portrayed in detail. 


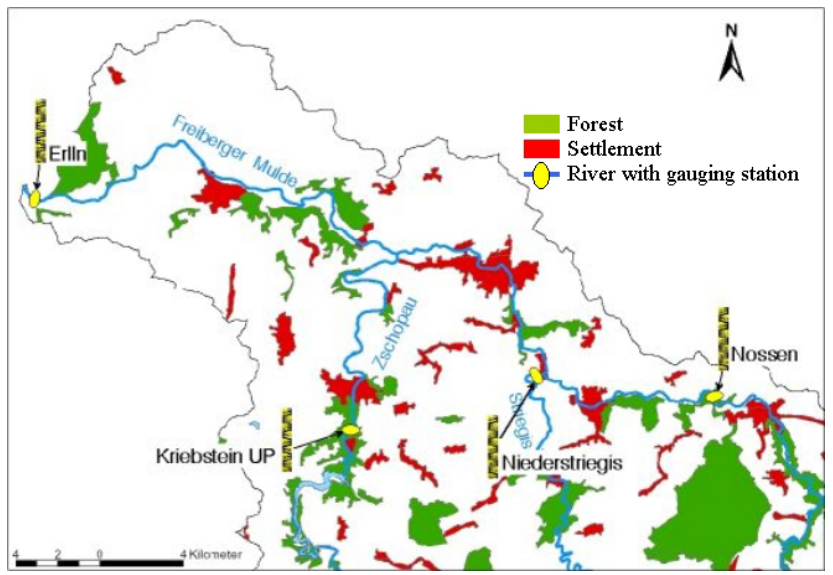

Fig. 1. Detail of the portrayed river sections.

\subsection{Hydrodynamic modelling}

The foundation of the setup of the hydrodynamic model (HEC-RAS, USACE1, 2002) are geometrical data of the river bed and the floodplains. Terrestrial measurements may not include floodplains and have to be complemented with data of a digital elevation model using GIS (ArcView) combined with the HEC-GeoRAS extension (USACE2, 2002). Once the cross sections are defined, the Manning values have to be assigned. For the main channel typical values according to river section characteristics as slope and width can be allocated. The Manning values of the floodplains correspond to land use and are assigned by means of HEC-GeoRAS. To obtain a sufficient resolution in space additional cross sections are interpolated.

Cross sections at gauging stations are appointed as upper boundary locations (see Fig. 1). A normal depth provides the lower boundary condition. To avoid interference with the target gauge, it has to be located far enough downstream of the target gauge. Internal boundary conditions represent lateral inflow from subcatchments alongside the modeled river sections.

For calibration and validation purposes the performance of the hydrodynamic model is evaluated by using historical flood discharge hydrographs. To calibrate the model, the Manning values have to be arranged to increase model performance. Because of a relatively high uncertainty concerning the rating curves at high water levels it seems evident that absolute discharge values are not very significant parameters for the evaluation of the model performance. Instead, the flow peak propagation time is a much more meaningful measure.

\subsection{Generation of the training data base}

After obtaining a validated model for the river system, the next step is to find relevant information for the training of

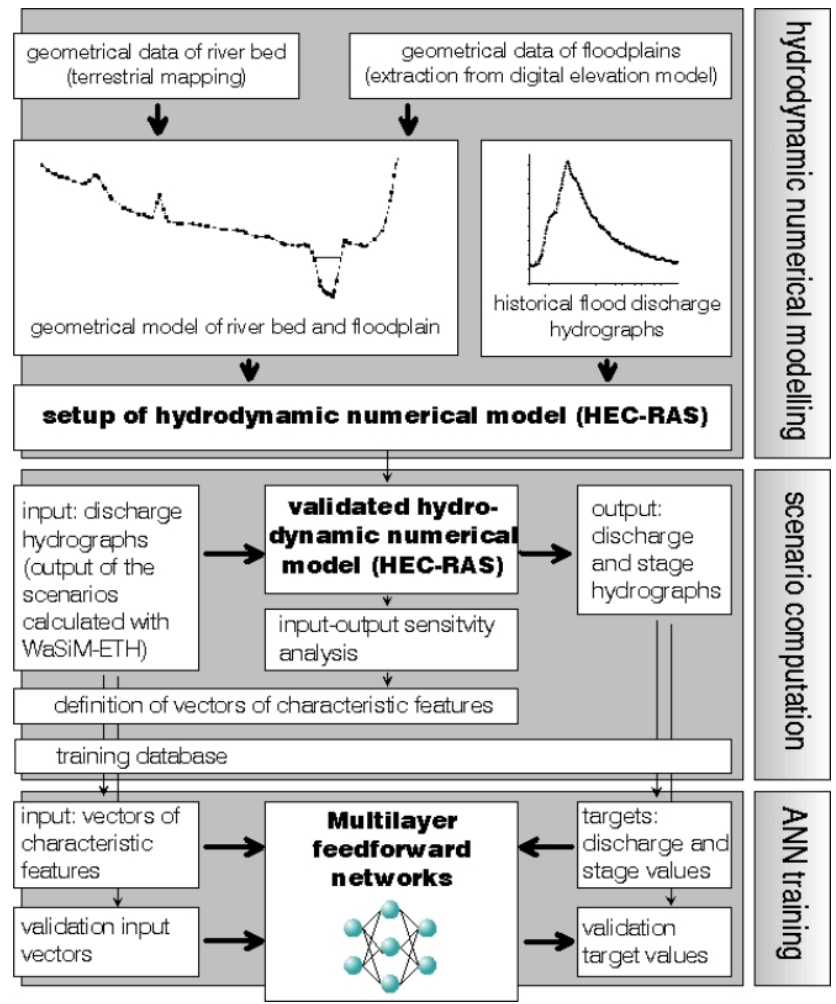

Fig. 2. Flow chart of the methodology.

an ANN. Before describing the applied network architecture and the consequential selection of vectors of characteristic features, we will explain some general properties of ANN. Since Artificial Neural Networks are black box models, they only got a restricted extrapolation ability. Therefore training a neural network requires sets of input and output data covering the whole range of possible flood events. Observed data will not accomplish this condition because continuous measurements are only available for a few decades. To get a more complete data set of possible flood events these are generated by applying the hydrodynamic numerical model. For the generation of realistic sets of flood discharge hydrographs as upper boundary conditions of the hydraulic model, a detailed analysis of the upstream catchment was performed. All relevant scenarios of weather situations containing the whole range of realistically possible flood events were used in a calibrated rainfall-runoff-model. By this way we get flood hydrographs at the upper limits of the modelled river reaches.

\subsection{Multilayer feedforward networks}

Multilayer feedforward networks (MLFN, also referred to as multilayer perceptrons) are simple but powerful and very flexible tools for function approximation. Figure 3 shows the basic structure of such a MLFN.

In general, MLFN map an input vector to an output vector (or single output value). The input vector must contain all 
the relevant variable informations for the reproduction of the network output. Therefore we will also refer to this input vector as vector of characteristic features. The proper choice of the values associating this input vector appears to be the most important step in setting up the MLFN. All the constant components of the modelling process like the cross section geometry are represented by the trained network as a black box system.

Such a network consists of several layers containing a number of neurons. The inputs to any neuron of the first layer are all the network inputs (normalised to between -1 and 1), at any other layer the neuron inputs are the outputs of the neurons of the precedent layer. The outputs of every neuron of the last layer are the network outputs. Therefore, it is called output layer. All the others are referred to as hidden layers.

A layer of neurons is determined by its weight matrix, a bias vector and a transfer function. The adjustment of the weight matrix and the bias vector is a matter of the network training, the transfer functions get predifined. Inside a hidden layer a sigmoid transfer function is applied which guarantees, that the layer outputs remain inside the range between -1 and 1 . The output layer applies a linear transfer function.

For this application a neural network with one hidden layer was used. In fact, MLFN with a single hidden layer can approximate virtually any function of interest to any degree of accuracy, provided sufficiently many neurons in the hidden layer are available (Hornik et al., 1989). The actual number of hidden neurons has to be estimated by trial and error. The number of neurons in the output layer equals the number of desired outputs.

For further reading on Artificial Neural Networks Hagan et al. (1996) can be recommended.

\subsection{Vector of characteristic features}

Refering to the previous paragraph, the focus on the setup of the MLFN is the choice of the input vector, i.e. the choice of the relevant features. To keep the problem as simple as possible the output is limited to a single value of flow discharge at a single cross section. (Likewise, this methodology has been applied for stage values too.)

The information relevant for a single target value at a downstream location consists of a finite number of discharge values at the upper boundary locations. The aimin appointing the vector of characteristic features is, in fact, to find all the upstream boundary discharge values that affect the target discharge value at the downstream location. Because of dealing with continuous time series, these discharge values are represented by time steps.

As a first step a sensitivity analysis of a single input value to the output hydrograph has been performed - by applying the hydrodynamic numerical model. The alteration of the discharge value at the timestep $t_{0}$ of a single upstream bound-

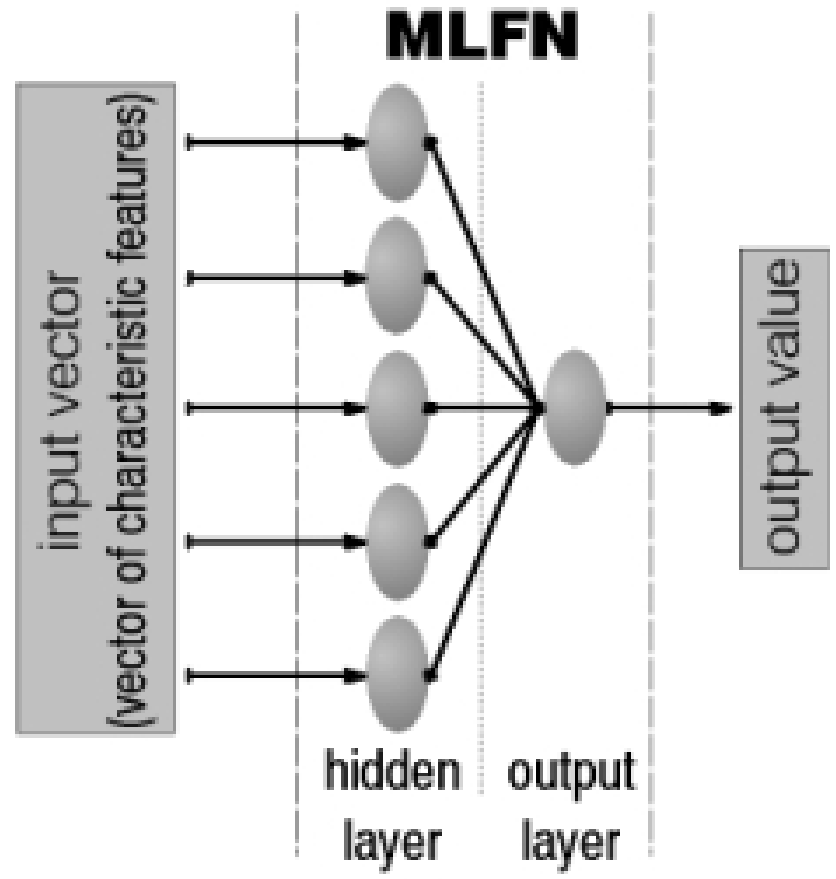

Fig. 3. Structure of the MLFN.

ary condition influences the output hydrograph between the time steps $t_{\min }$ and $t_{\max }$ (Fig. 4).

If $t_{0}$ stands for time step number zero, the indices min and max represent the numbers of the time steps, between which the alteration of the input value shows an effect at the model output.

$Q\left(\right.$ input, $\left.t_{0}\right)->Q\left(\right.$ output, $\left.t_{n}\right), \min \leq n \leq \max$

$Q$ (input, $\left.t_{1}\right)->Q$ (output, $\left.t_{n}\right), \min +1 \leq n \leq \max +1$

$\ldots$

In reverse, assuming that there is only one upstream boundary condition, a single output value at a certain time step $t_{0}$ is only influenced by the input values at the time steps between $t_{(-\max )}$ and $t_{(-\min )}$.

$Q\left(\right.$ input,$\left.t_{n}\right),-\max \leq n \leq-\min ->Q\left(\right.$ output,$\left.t_{0}\right)$

Or:

$Q\left(\right.$ output, $\left.t_{0}\right)=f\left(Q\left(\right.\right.$ input,$\left.\left.t_{n}\right),-\max \leq n \leq-\min \right)$

That means, that the output value is a function of the input values dating back between $t_{\min }$ and $t_{\max }$ (Fig. 5). This methodology has to be applied for every upstream boundary location. The identified input values are the characteristic features for the output values (case 1).

Furthermore $t_{\min }$ is identical with the forecast horizon $t_{\text {for }}$. So it is reasonable to augment this value, if the consequetial 


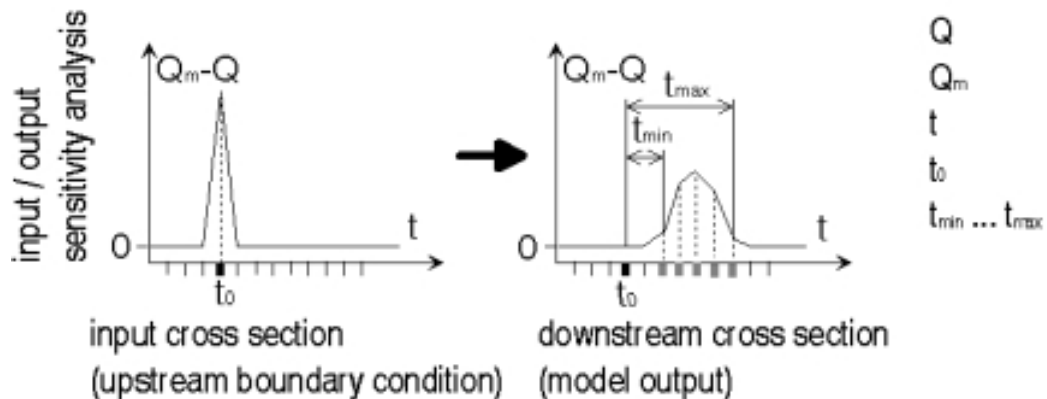

\author{
discharge \\ discharge, alterated \\ time \\ time step of input value alteration \\ time span, in which an alteration at \\ the input hydrograph shows an effect \\ at the target hydrograph
}

Fig. 4. Sensitivity of the the output hydrograph to a single input value.

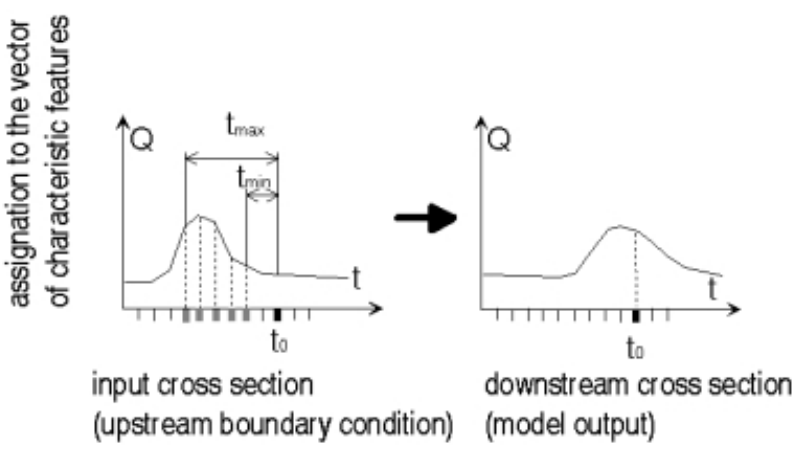

Fig. 5. Assignation of time spans to the input vector.

loss in accuracy remains small. The number of characteristic features decreases, and certainly the performance will decline too. To which extend $t_{\text {for }}$ can be increased, depends on the specific catchment, but certainly $t_{\text {for }}$ must not exceed the flow peak propagation time of the main tributaries (case 2).

\section{Study area and data}

The Mulde catchment $\left(2983 \mathrm{~km}^{2}\right)$ at Erlln gauge, in the Ore mountains (Erzgebirge, East Germany) serves as a first test application of this new methodology. The elevation stretches between some $1200 \mathrm{~m}$ and $140 \mathrm{~m}$ at the outlet. The land use mainly consists of forest (30\%), agricultural land (47\%), the remainder being urbanised terrain and fallow. It was heavily affected by the flood of August 2002.

The river reaches in the study area (Fig. 1, Table 1) amount to more than $60 \mathrm{~km}$ including parts of the Zschopau and Striegis rivers. 551 cross sections with a mean distance of 120 metres characterise the river bed and the valley. The cross sections are extracted from a digital elevation model $(20 \mathrm{~m} * 20 \mathrm{~m} * 0.1 \mathrm{~m}$ resolution) combined with data of a terrestrial survey from the late sixties.

The upper boundary conditions consist of flow hydrographs at the inflow gauges, as described in Table 1. As mentioned above, the lower boundary condition must not in- fluence the target cross section at the Erlln gauge. Therefore, a constant friction slope some $8 \mathrm{~km}$ downstream of Erlln was appointed.

The discharge hydrographs at the upstream boundaries represent about 90 percent of the total area at the outlet. The remaining subcatchments are not equipped with gauging stations. To estimate their influence to the total discharge we applied a rainfall-runoff-model with regionalised parameters.

The availability of discharge data with a resolution of one hour is within the scope of expectancy. The mentioned flood of 2002 resulted in a destruction of several gauging stations, amongst them the gauging station at Erlln. The highest stages recorded at every of the four gauges within the study area date back to December 1974. This flood event was used for validation purposes.

\section{Results}

The flow discharge hydrograph calculated by the hydrodynamic model for the validation flood of 1974 (Fig. 8) shows a good reproduction of the measured one. Rating curves have considerable uncertainties in cases of extreme floods. That is the reason why in these cases absolute values are not very trustworthy. But criteria like the shape and the flood peak propagation time prove to be a good model of the natural system.

The hydrodynamic numerical modelling regarding geometrical parameters and the distance between the cross sections requires computation intervals of some 15 seconds. For the generation of the data base of possible extreme flood events time series of 20 years had to be computed.

The application of this methodology for the estimation of the input vector of the MLFN produces a result as shown in Table 2. The internal boundary conditions representing the lateral inflow are not independent from the other input hydrographs and the respective subcatchments are relatively small compared to the total catchment. Due to this they are not represented in the input vector of the MLFN. The dots on the hydrographs of the upstream gauges in Fig. 6 symbolise the characteristic features for the single output value at the 
Table 1. Catchment characteristics of the study area.

\begin{tabular}{llcc}
\hline Upper boundaries: & & \\
\hline Gauge & Tributary & Area $\left[\mathrm{km}^{2}\right]$ & Mean flow $\left[\mathrm{m}^{3} / \mathrm{s}\right]$ \\
\hline Kriebstein UP & Zschopau & 1757 & 24 \\
Nossen & Freiberger Mulde & 585 & 7 \\
Niederstriegis & Striegis & 283 & 2.7 \\
\hline Lower boundary: & & \\
\hline \multicolumn{4}{l}{ Constant friction slope some 8 km downsteam of the target gauge at Erlln } \\
\hline
\end{tabular}

Table 2. Propagation times to the Erlln gauging station.

\begin{tabular}{lcc}
\hline Gauge & Peak propagation time & Influence interval \\
\hline Kriebstein & $6 \mathrm{~h}$ & $3-9 \mathrm{~h}$ \\
Nossen & $8 \mathrm{~h}$ & $6-9 \mathrm{~h}$ \\
Niederstriegis & $6 \mathrm{~h}$ & $6-7 \mathrm{~h}$ \\
\hline
\end{tabular}

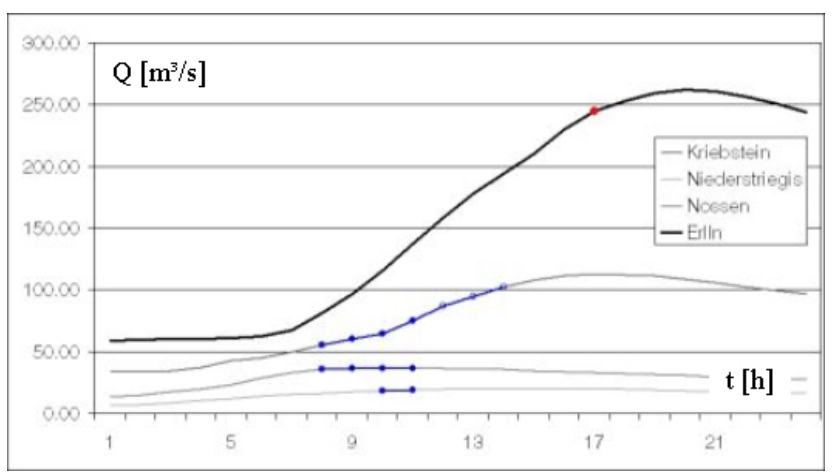

Fig. 6. Characteristic features (blue dots) for the target discharge value (red dot).

target hydrograph (case 1). Reducing this set by the unfilled dots keeps only values within a distance of at least $6 \mathrm{~h}$ (minimum flood peak propagation time) to the target value (case 2).

For both cases MLFN with 15 neurons in the hidden layer were trained. Figure 7 shows a comparison of the network outputs and the outputs of the hydrodynamic model (herein referred to as targets) as normalised values. The mapped data have not been used as training data. In both cases an excellent performance of the trained MLFN can be reported.

Figure 8 compares the output of the MLFN (case 2) with the output of the hydrodynamic model and the measured hydrograph for the 1974 flood event. The trained MLFN is able to reproduce the performance of the physically based model to a satisfying degree of precision.

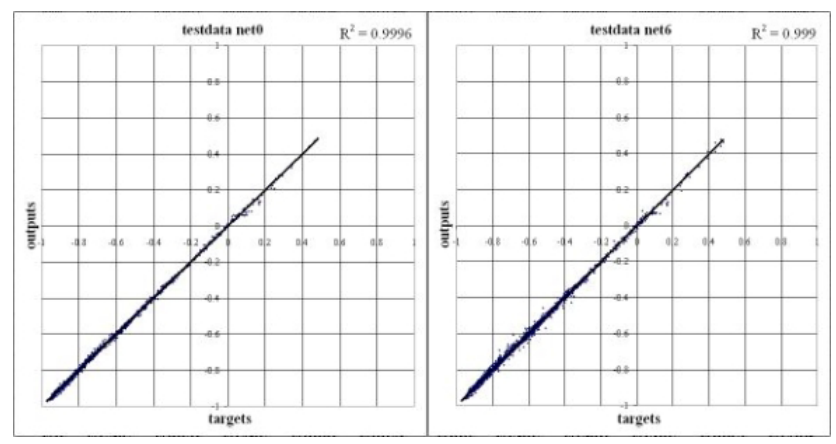

Fig. 7. Correlation of MLFN output with output of HEC-RAS, left: case 1, right: case 2).

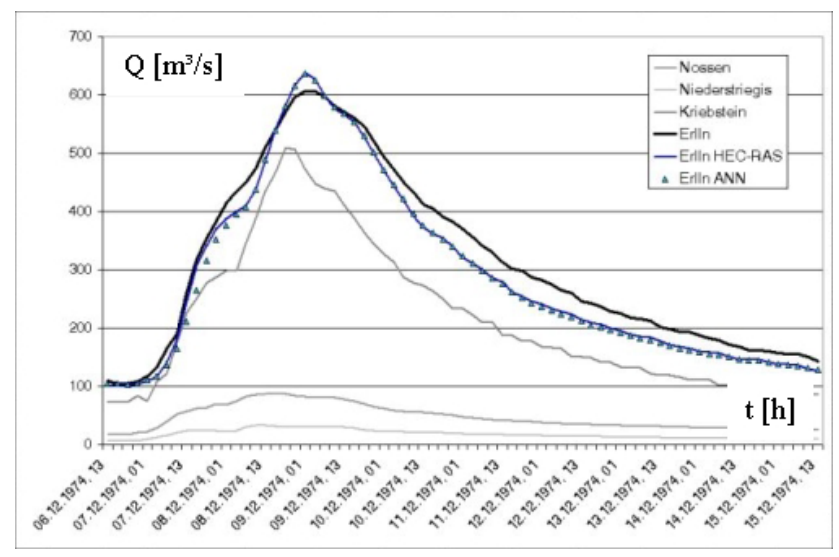

Fig. 8. Performances of HEC-RAS and the MLFN for the flood event of 1974 .

Moreover, the application of the MLFN is much faster than HEC-RAS. The calculation of a time series of one year takes less than a second, compared to about $12 \mathrm{~h}$ with the hydrodynamic numerical model. 


\section{Conclusions}

This contribution presented a new methodology to combine hydrodynamic numerical modelling with artificial intelligence. The goal is to overcome both the restricted extrapolation capabilities of artificial neural networks and the high computation requirements concerning the application of sophisticated physically based modelling. The advantages of the use of ANN for flood prediction purposes have been emphasised. Notwithstanding, the ability of a hydrodynamic model to deal with extreme floods beyond recorded events has been incorporated.

Moreover, the hydrodynamic numerical model is an appropriate tool to find the characteristic features for the input vector of the MLFN. This is the basis for an efficient utilisation of the input information.

In fact, the trained MLFN reproduces the model performance in an excellent manner. The advantages of the use of artificial intelligence are obvious: A noticeable decrease of computation time may be useful for on-line flood forecasting. Furthermore, by reducing the vector of characteristic features the forecast horizon can be increased up to the flow peak propagation time.
Edited by: R. Barthel, J. Götzinger, G. Hartmann, J. Jagelke, V. Rojanschi, and J. Wolf

Reviewed by: anonymous referees

\section{References}

Hagan, M. T., Demuth, H. B., and Beale, M.: Neural Network Design, PWS Publishing Company, Boston, 1996.

Hornik, K. M., Stinchcombe, M., and White, H.: Multilayer feedforward networks are universal approximators, Neural Networks, 2, 5, 359-366, 1989.

Minns, A. W. and Hall, M. J.: Artificial neural networks as rainfallrunoff models, Hydrol. Sci., 41, 399-417, 1996.

Shrestha, R. R., Theobald, S., and Nestmann, F.: Simulation of flood flow in a river system using artificial neural networks, Hydrol. Earth Syst. Sci., 9(4), 313-321, 2005.

USACE1: US-Army Corps of Engineers, HEC-River Analysis System. Hydraulic Reference Manual, Version 3.1, http://www.hec. usace.army.mil/software/hec-ras/, 2002.

USACE2: US-Army Corps of Engineers. HEC-GeoRAS, An extension for support of HEC-RAS using ArcView, http://www.hec. usace.army.mil/software/hec-ras/, 2002. 